\title{
Le Nkisi dans la tradition woyo du Bas-Zaïre
}

The Nkisi in the Woyo traditions of Lower Zaire

\section{Mulinda Habi Buganza}

\section{OpenEdition}

Journals

Édition électronique

URL : http://journals.openedition.org/span/1070

DOI : $10.4000 /$ span. 1070

ISSN : 2268-1558

\section{Éditeur}

École pratique des hautes études. Sciences humaines

\section{Édition imprimée}

Date de publication : 1 septembre 1987

ISSN : 0294-7080

\section{Référence électronique}

Mulinda Habi Buganza, "Le Nkisi dans la tradition woyo du Bas-Zaïre », Systèmes de pensée en Afrique noire [En ligne], 8| 1987, mis en ligne le 07 octobre 2013, consulté le 30 avril 2019. URL : http:// journals.openedition.org/span/1070 ; DOI : 10.4000/span.1070 


\title{
LE NKISI DANS LA TRADTTION WOYO DU BAS-ZAIRE 1
}

\author{
par
}

\section{Mulinda Habi Buganza}

Les minkisi (sg. nkisi) des Kongo ont été depuis quelques années l'objet de plusieurs travaux écrits par des anthropologues et des historiens de l'art africain2. Ces études, généralement basées sur les écrits du missionnaire suédois Karl Laman et complétées, en ce qui concerne les auteurs américains, par les commentaires d'un informateur privilégié, Fu-kiau Kia Bunseki, décrivent les minkisi comme appartenant à un système de pensée commun à tous les groupes kongo et relevant d'un unique modèle d'interprétation. Force est cependant de constater qu'il existe plusieurs interprétations de ce phénomène culturel, et que le modèle proposé par ces auteurs est fortement influencé par la conception religieuse particulière au groupe qu'ils étudient sur le terrain.

En effet, s'il est généralement admis que le nkisi est un objet ou un ensemble d'objets auquel est attribuée une force surnaturelle, les interprétations de la nature et du mode d'action de cette force varient sensiblement suivant les groupes kongo. Alors que le nkisi est considéré chez les Yombe comme une "force universelle et immatérielle" (Doutreloux, 1967:225), les Ntandu et les Ndibu pensent qu'il s'agit de "I'esprit (d'un mort) dominé par l'homne" (Van Wing, cité par Hagenbucher-Sacripenti, 1973:104) ou encore d'un

\footnotetext{
${ }^{1} \mathrm{Ce}$ texte est tiré de notre dissertation doctorale présentée le 18 décembre 1985 à I'Université de Bruxelles.

2Hirschberg 1971, Volavka 1972 et 1974, MacGaffey \& Janzen 1974, Dupré 1975, MacGaffey 1977 et Jacobson Widding 1979.
} 
"esprit de la nature" (Buakasa Tulu, 1973), tandis que les Vili croient que le nkisi est une incarnation de l'esprit d'un membre du clan (Hagenbucher-Sacripenti, 1973:104). Par ailleurs, alors que pour certains le nkisi est lié à la sorcellerie et donc considéré comme foncièrement mauvais, pour d'autres c'est une force neutre sinon utile aux individus et aux groupes dont il assure la protection.

Ces différences portant sur la nature du nkisi, relevées dans des études relatives à des cultures particulières, prouvent combien grand est le risque de tomber dans des généralisations faciles et des synthèses prématurées tant qu'on ne dispose pas de données suffisantes sur la majorité des sous-groupes kongo. Nous nous limiterons donc ici à l'examen de la conception du nkisi chez les Woyo. Un missionnaire du 17ème siècle présente ce peuple comme "adonné à la sorcellerie et à la magie" (Merolla da Sorento, 1702: 717). Un autre affirme au début de ce siècle que les Woyo sont connus comme "spécialistes de certains fétiches, et pour leur religion particulière" (Bittremieux, 1934: 204). Nous évoquerons brièvement le cadre géographique, historique et religieux woyo; nous examinerons ensuite la nature et le contenu des minkisi, leurs fonctions sociales et religieuses, leur rapport à la sorcellerie.

\section{Les Woyo, pays et culture}

Les Woyo, qui constituent un sous-groupe de l'ensemble culturel kongo, habitent un territoire délimité à l'ouest par l'océan Atlantique, au sud par l'embouchure du fleuve Zaïre, à l'est, au Zaïre, par la rivière Mbola, et au nord, dans la province angolaise du Cabinda, par la rivière LuIonda. Les données que nous allons exposer ont été récoltées dans la partie zaïroise connue sous la dénomination administrative de "collectivité de la Mer"3 comprenant plus d'une cinquantaine de villages situés pour la plupart le long d'axes routiers.

A partir du littoral, ce territoire se présente comme une succession de plateaux au sol sableux couverts d'une savane herbeuse

3La "Collectivité de la Mer" fait partie de la Zone de Muanda, Sous-région de Boma, Région du Bas-Zaîre. 
contrastant avec quelques zones boisées situées le long des cours d'eau. Ce paysage se transforme en une forêt dense au fur et à mesure qu'on s'éloigne de la côte. Le climat est subéquatorial, négativement influencé par le courant froid de Benguela, si bien qu'une grande partie du territoire est caractérisée par une longue saison sèche et une pluviométrie faible; ce qui explique les nombreuses famines, rapportées par plusieurs témoins du siècle dernier (De Rouvre 1880:295; De Mattos de Siva 1904:323; Dennett 1887:11), et que les woyo interprètent comme un fléau envoyé par les esprits de la terre pour sanctionner la rupture d'un interdit.

C'est l'aventurier anglais Battell qui le premier, au début du 17 e siècle, décrivit le territoire des Woyo, qui formait alors le royaume de Ngoyo. Entouré de deux puissants voisins, les royaumes de Loango et de Kakongo, ce petit état nous est présenté comme vassal du premier. A la fin du siècle, le Hollandais Dapper nous apprend qu'il était devenu indépendant et que son port, Cabinda, jouait un rôle important dans le commerce transatlantique. Dès le XVIIIe siècle, Ngoyo prit une part active dans la traite des Noirs et devint le principal fournisseur d'esclaves de la Côte au sud de I'Equateur. Cet état-courtier ne put survivre aux abolitions successives de l'esclavage. Sa chute fut définitive en 1885 lorsque le territoire de Ngoyo fut partagé entre le Portugal et la Belgique.

La disparition du royaume de Ngoyo donna lieu au développement de l'organisation sociale actuelle, une structure matrilinéaire dominée par l'autorité du chef de clan. Ce dernier assure la gestion des affaires du clan et est responsable du bien-être de chaque membre, assumant à la fois des fonctions sociales, juridiques et religieuses.

Les Woyo croient en l'existence de plusieurs catégories d'êtres surnaturels dont la hiérarchie est la suivante: un Etre suprême, plusieurs esprits de la terre (bakisi ba si, sg. nkisi si), des génies (simbi), les esprits protecteurs des familles ou des individus (tungu), ainsi que les esprits des morts, dont les plus importants sont les ancêtres (bakulu). Tous ces esprits sont considérés comme des entités individuelles et personnalisées; les esprits de la terre regoivent cependant, dans des sanctuaires qui leur sont dédiés, 
un culte collectif célébré par un corps hiérarchisé de dignitaires religieux (un chef, un ou plusieurs prêtres, des assistants et les membres des associations cultuelles, notamment l'association des masques ndunga). Au niveau de chaque clan par contre, il existe un culte des ancêtres célébré par le chef de clan. Ces ancêtres sont généralement des personnalités du clan (hormes et femmes) qui ont joué un rôle éminent; ils sont supposés séjourner dans un au-delà situé dans le monde souterrain, qui se trouve être aussi symbolisé par la forêt. Sont exclus de cet univers tous ceux qui n'ont pas observé de leur vivant les prescriptions des ancêtres; livrés à eux-mêmes, ces morts deviennent des esprits errants, minkulu, que certaines personnes peuvent capter dans le but de renforcer la puissance de leurs minkisi, à moins qu'ils ne se transforment en êtres fantomatiques et évanescents qui, en désespoir de cause, se réfugient dans certains animaux et disparaissent ainsi du monde humain sans pour autant disparâtre du circuit de la vie.

\section{Nature et contenu des minkisi}

Les minkisi forment la dernière catégorie des phénomènes surnaturels auxquels croient les Woyo. Ils sont considérés comme des forces surnaturelles indéterminées et impersonnelles qu'une personne (nganga nkisi) habilitée à les manipuler peut enfermer dans des supports matériels qui de ce fait sont aussi appelés minkisi, marquant ainsi une superposition de l'objet et de sa représentation.

Une des caractéristiques du nkisi est son ambivalence : c'est une force qui peut être utilisée aussi bien pour des actions d'attaque que pour annuler des intentions agressives. Cette force est au service d'un propriétaire (nganga nkisi), qui la met à la disposition de ses proches et de sa clientèle. Le nkisi, cependant, n'est pas lié à la personne du nganga car, dit-on, nganga yafwa nikis ikele, "le nganga peut mourir, le nkisi demeure".

La forme du nkisi revêt une grande importance car elle détermine son mode d'action. Elle dépend du support matériel choisi comme réceptacle des éléments symbolisant la puissance du nkisi. Les 
réceptacles traditionnels les plus courants sont les statuettes en bois sculpté (ndubi), avec un ou deux miroirs (mbandi), des poteries (kombe), des paniers (ntende), des corbeilles (tanda), des cornes (mpoka), des coquilles (nkole), et des paquets ou sachets (lifuta). Actuellement on trouve aussi des bouteilles de toutes dimensions et des boites de conserve. Le réceptacle le plus simple est un paquet en tissu bleu ou en raphia contenant les éléments symboliques communs à presque tous les minkisi. Ces éléments sont d'origine minérale, animale ou végétale.

Les éléments d'origine minérale les plus usités sont les suivants: une sorte de caillou beige (lenjye) ramassé au bord de la mer dont la compacité et la solidité symbolisent la résistance à toute épreuve; une roche friable et blanche (tondo), et le kaolin (mpezo) dont la couleur blanche représente les chances de réussite dans toute entreprise ; de la vase provenant du fond d'un étang ou du lit d'une rivière, ou encore de la terre d'un cimetière, qui évoquent ce monde souterrain qu'est l'au-delà.

Parmi les éléments d'origine animale figurent les coquilles des mollusques liseve et kongolo, qu'il faut extraire avec précaution sans quoi ils provoquent des maux de ventre aux consommateurs imprudents; ta nsoso et nzinga, deux autres bestioles de la mer, qui traînent patiemment des coquilles énormes pour leurs corps ; les arêtes du poissons-scie électrique, dont les réactions sont brusques et dangereuses. On trouve aussi des parties d'animaux terrestres comme le rongeur simbizi, animal difficile à débusquer, ou le porc-épic (ngumba) qui en hérissant ses piquants se rend inattaquable; ou encore une dent de porc, lequel pour se nourrir doit fouiner partout, une patte d'un animal arboricole appelé tshinkanda dont on dit qu'il ne lâche jamais sa proie, une écaille de pangolin (nkaaka), qui a la propriété d'éloigner les actions agressives des autres minkisi en vertu du principe que traduit le proverbe suivant: ngandu ka mina nkaaka, nkaaka ka mina ngandu, kali ya kali bapodi mimena ko, "le crocodile n'avale pas le pangolin, le pangolin n'avale pas le crocodile, les animaux à écailles ne s'avalent pas entre eux." On trouve aussi dans les minkisi des serres d'aigle, oiseau que son vol élevé rend inaccessible et dont les serres ne lâchent jamais leur 
proie, et certains organes appartenant à des oiseaux nocturnes qui ont la faculté de voir sans être vus. Enfin, des morceaux du nid de l'insecte ntebitshye (qui se fixe n'importe où) et de la chenille piquante fukalili.

Les éléments végétaux apparaissent souvent en association, notamment : ntumbu Mvemba, thiala mioko et funda nkata. Ntumbu Mvemba (Dioclea flambellipes, papilionaceae) est une graine légèrement discoïde et dure qu'on trouve à l'embouchure du fleuve Zaïre, lieu placé sous la tutelle de l'esprit de la terre Mvemba. Dépourvue d'utilité pratique, elle symbolise la concorde et l'entente basées sur la sincérité. Tshiala mioko (Schrebera golugensis, olaceae ou Leea guineensis G. Don, Leeaceae) est une graine qu'on trouve sur la plage. Son nom signifie "ouvre les mains"; sa forme suggère en effet deux mains ouvertes ou tendues. Elle symbolise la générosité, la disponibilité et l'ouverture d'esprit, qualités que recommande ce proverbe, qui lui est lié : tshiala miolo ke zifumu ke zinganga, ke babonso, "tends les mains (montre-toi disponible) aux chefs, aux sages, à tout le monde". Funda nkata, enfin, est aussi une graine qui provient de la plage; elle est ronde et plate, et sa forme rappelle la position en tailleur (funda zinkata), posture de respect. Le proverbe qui lui est lié est le suivant : funda zinkata ke zifumu ke zi nganga, "sois respectueux envers les chefs et les sages". Ces trois graines évoquent le discernement qui doit accompagner la mise en oeuvre du nkisi : une faute dont on s'est repenti n'est plus punissable et les gens honnêtes sont présumés innocents.

On trouve aussi, enfoncées dans une pâte de tukula (poudre rouge) plusieurs sortes de plantes : 1) malemba mpumbu (brillantaisia patula acanthaceae), dont la vertu médicinale est de calmer (lemba signifie calmer) ; 2) matelaka ngolo (clero dendron volubilis Beauv. verbenaceae) dont le nom signifie littéralement "qui relève l'énergie", et nduli nduli masala ma nkusu (clerodendron splendens G. verbenaceae), littéralement "l'amer aux couleurs du perroquet", deux plantes connues par leur amertume; 3) bulukutu (occimum ratissimum Linn. Latitae) dont l'odeur est répugnante pour certains ; 4) lolo (annona senegalensis Pers. annonaceae), arbuste dont les feuilles touffues constituent une cachette idéale et qui résiste 
aux incendies de brousse ; 5) batabata (boerrhavia diffusa Iinn. Nyctagynaceae), de batama, "couvrir", plante qui recouvre et étouffe tout sur son parcours ; 6) nkasa (erythrophleum guineense Don Leg) qui était utilisée pour les épreuves de poison ; 7) nyoka nyoka (cassia occidentalis Linn caesalpinaceae), plante vermifuge censée éloigner les serpents ; 8) mobolo (cissus aralioides Welw ex Bak. vitaceae) liane qui ressemble à une espèce de serpent local ;

9) zika zika (eleusine indica Gaetrn gramineae), graminée qui pousse sur les sentiers provoquant la chute des marcheurs inattentifs ; 10) nsanga valu (costus afer Ker zingiberaceae) dont il existe plusieurs usages dans les rituels traditionnels 4.

Il importe de constater que le symbolisme véhiculé par tous ces éléments n'implique en aucun cas l'agressivité ; il s'agit au contraire de donner l'impression que les puissances "embusquées" dans le nkisi protègent son propriétaire, dissuadant ainsi l'agresseur potentiel. Autrement dit, le choix de ces éléments ou leur association doit orienter l'action d'un nkisi non pas vers l'attaque mais vers la parade et la riposte. On comprend dès lors pourquoi, comme nous le verrons, le propriétaire d'un nkisi doit simuler une action agressive (planter un clou, par exemple) tout en désignant au nkisi la victime sur laquelle doit s'exercer la riposte.

Particularités des minkisi en fonction de la forme des réceptacles

Après une sélection guidée par le souci de l'efficacité, les éléments symboliques du nkisi sont conservés dans un sachet ou enveloppés dans un paquet en coton ou en raphia. Ce paquet peut en lui même constituer un nkisi, notamment pour garantir le pouvoir thérapeutique d'un devin, mais il est généralement introduit dans un autre support pour constituer l'élément essentiel du nkisi appelé ntima, son coeur, ou longo (milongo), médecine. Le paquet peut ainsi être placé dans une corbeille (tende) ou un panier (ntende). Le

4La plupart de ces végétaux sont des plantes médicinales. A cette liste, il faut ajouter la fleur de la graminée nkote (non identifiée) et l'écorce de l'arbre budunji (non identifié). 
nkisi représente alors le pouvoir thérapeutique de certains guérisseurs ; dans certains cas, le nkisi n'a de pouvoir que sur une maladie spécifique (par exemple les minkisi Maluangu Songo et Kalunga agissent sur les maladies féminines).

Si le paquet est enfoui dans l'argile contenue dans un pot, le nkisi porte le nom de mbumba (de bumba modeler, pétrir). Bumba, dont on dit qu'il vient des Bayombe, est un nkisi qui favorise la chance, la richesse et autre bonne fortune, et assure la longévité (lors des guerres par exemple, les guerriers devaient le toucher pour se rendre invulnérables).

L'activité du nkisi mbumba est entretenue par une eau lustrale régulièrement versée dans le pot. Dans certains cas, cette opération sert à la divination : on observe le temps que met l'eau à disparấtre; si elle disparait rapidemert la situation pour laquelle on consulte peut être considérée comme désespérée. Ajoutons que le nkisi mbumba est une force redoutable car il implique la mise à mort par sorcellerie d'un ou plusieurs membres de la famille, dont le principe vital doit servir à son activation et à son animation. La victime idéale est une personne occupant la position de neveu (nièce), dont le comportement a été jugé insupportable ou incompatible avec les normes sociales; les proies les plus faciles appartiennent, semble-t-il, aux sections les plus faibles du clan, celles qui comportent peu d'hommes; il peu également s'agir d'homme de peu de poids social. On comprendra donc que le propriétaire du nkisi mbumba peut être considéré comme sorcier.

Les statues (ndubi, zindubi, de forme et de taille variable), constituent une catégorie importante de minkisi. Elles sont de plusieurs sortes : les minkisi de divination (lumonya mpiya) munis d'un seul miroir ventral; les minkisi de protection (mbandi) avec deux miroirs (dorsal et ventral); les minkisi d'imprécation (nkisi loko) qui regoivent les charges d'activation dans la région ombilicale.

Le nkisi de divination est généralement de petite taille. Son miroir ventral (ou logé dans les cavités oculaires) est censé lui accorder une vision interne des réalités cachées. Le devin I'utilise, avec d'autres techniques divinatoires, pour s'assurer que toutes les causes des maladies et des malheurs ont été découvertes. 
Le nkisi de protection est une statue de bois anthropomorphe dont les miroirs ventral et dorsal représentent la capacité de détecter les intentions ou les actes d'agression, dans le but de les neutraliser et de les détruire 5 .

Les minkisi d'imprécation sont de grande dimension, parfois de taille humaine: qu'ils soient des représentations humaines ou animales, ils ont souvent une attitude menaçante afin d'éloigner les mauvais esprits (nkolu ou nkuyu) et surtout les sorciers (ndotshi). Les représentations humaines sont souvent coiffées d'un couvre-chef en raphia, orné d'une pointe ou de plumes ; elles sont pourvues d'une barbe, habillées d'un pagne indigo posé en toge ou retenu à la taille par une grosse ceinture. Les orbites sont obstruées par des miroirs, ce qui leur donne un aspect terrifiant. Un miroir peut aussi être installé au ventre, retenu par du copal, bouchant l'orifice où sont logés les ingrédients constitutifs de la puissance du nkisi. On pense que le propriétaire y enferme le principe vìtal d'un ou de plusieurs membres de son clan, d'un esclave ou encore d'un esprit errant en vue d'activer la puissance du nkisi. Le propriétaire du nkisi loko est donc un personnage redouté.

Chaque nkisi imprécatoire portait autrefois un nom dont la réputation dépassait les frontières woyo. Parmi ces noms, on peut citer Mabiala, Ndombe, Mpizi, Mavungu, Tshinshimuna, Suma, Mangaka, etc., qui sont des statues à forme humaine, et Kozo, statue-Janus représentant deux chiens prêts à mordre 6 . Dennett (1901-1902) rapporte que les plus réputés à son époque (1870-1890) étaient Mabiala (Ma Ndombe), Mavungu et surtout Mangaka dont le souvenix est encore vivace à Muanda (cf. aussi Delange 1967: 137-138). Dans ce village, Mangaka nous a été décrit comme une statue en bois anthropomorphe de $1 \mathrm{~m} 40$, portant une ceinture frangée retenant aux hanches un pagne indigo et tenant dans la. main droite un couteau menaçant, le bras droit collé le long du corps. Les yeux obstrués par deux miroirs, la barbe

5 Statues à ne pas confondre avec les statues féminines d'ancêtres. Les. unes et les autres ne sont plus en usage chez les woyo.

${ }^{6}$ Le nom générique n'konde que leur donnent les critiques d'art africain $n^{\prime}$ est pas connu chez les Woyo. Au mot -konde, ces derniers donnent le sens de "filet, toile d'araignée". 
collée au menton par une résine (bwangu), elle a un miroir dans chaque oeil, un troisième miroir au niveau du nombril obstruant une cavité servant de réceptacle. Mangaka porte aussi le nom de Mbwaca ("ce qui tombe, ce qui surprend") parce que, dit-on, il ne rate jamais la victime qu'on lui désigne. Aussi était-il craint dans toute la région. On lui prêtait le pouvoir de se déplacer, mais il était généralement porté sur une chaise longue par quatre personnes précédées par un sonneur de gong (ngonjye). Des clochettes attachées à ses oreilles servaient à signaler sa présence afin que les habitants l'évitent.

\section{La fonction des minkisi}

a. L'acquisition d'un nkisi

Plusieurs raisons peuvent conduire une personne à acquérir un nkisi. Ce sont principalement : la recherche de la guérison ou d'une protection contre les maladies 7 pour les minkisi thérapeutiques, la protection de la famille contre les attaques extérieures, le désir de richesse et de longue vie pour le nkisi mbumba, l'intention de réparation d'un tort ou la vengeance pour les minkisi imprécatoires.

L'acquisition d'un nkisi se fait de plusieurs façons : par héritage (généralement des parents maternels), par achat, mais plus souvent à travers une initiation. Celle-ci suppose une longue préparation. Elle se fait auprès d'un propriétaire de nkisi, le plus souvent un spécialiste, qui le nom de son nkisi comme titre. On choisit de préférence un étranger à la société woyo, à cause des retombées sociales et des implications de sorcellerie auxquelles nous faisions allusion. A part les minkisi thérapeutiques, qui n'impliquent aucun "sacrifice", les minkisi sont confectionnés dans le plus grand secret.

Après avoir fréquenté le spécialiste, le candidat cherche le support du nkisi qu'il veut acquérir : un pot neuf pour le mbumba, une statue pour les minkisi de divination et d'imprécation... Dès que les connaissances concernant l'usage du nkisi sont jugées suffi-

7 Autrefois il existait pratiquement un nkisi pour chacune des maladies courantes dans la région. 
santes, on procède à l'installation des ingrédients choisis parmi Ie matériel dont il a été question plus haut. Le pot (nzungu mbumba) est enveloppé dans un tissu d'étoffe indigo (bundi) ou dans une peau du reptile mbambi (probablement un varan) ou du rongeur nkumbi (une sorte de grand rat), deux animaux effrayants mais inoffensifs pour I'homme; ceci signifie que le nkisi est destiné à protéger le propriétaire et sa clientèle ; il est censé éloigner les malveillants sans les tuer.

L'initiation aux minkisi divinatoires se termine par le placement des ingrédients dans l'orifice ventral et son obstruction par du copal (linji) ou de la résine (bwangu) retenant un miroir. Pour les minkisi imprécatoires, il faut au préalable sacrifier une personne du clan au moment du choix de l'arbre à sculpter, en citant clairement le nom de la victime. La statue terminée, les ingrédients d'activation sont placés dans les orifices prévus. L'initiateur immole une poule dont le sang est versé sur les yeux, les narines, la bouche, la cavité ventrale puis au pied du nkisi afin, dit-on, de lui donner le goût du sang, ce qui fait du nkisi une puissance cannibale, devant poursuivre les ennemis des consultants jusqu'à leur élimination définitive. La confection d'un nkisi mbumba exige aussi un sacrifice sanglant.

\section{b. La consultation du nkisi}

La consultation du nkisi se fait pour les mêmes raisons que celles qui ont présidé à sa création. Ainsi le consultant d'un nkisi mumba se présentera-t-il chez le propriétaire pour se prémunir contre un danger imminent (chômage, prison, etc.), pour satisfaire ses ambitions, pour obtenir la richesse, le pouvoir ou une longue vie. Il apportera des rognures de ses ongles, une touffe de cheveux, des poils du pubis et des aisselles ainsi que de la poussière d'un lieu où il a placé ses pieds ou ses fesses. Ces éléments sont placés dans le pot de nkisi mbumba tandis que le spécialiste confie au consultant un petit paquet d'étoffe indigo contenant des morceaux de plusieurs éléments de mbumba, qu'il devra porter sur soi en permanence.

S'il s'agit de conjurer un danger imminent ou d'obtenir certaines grâces, le consultant est mis en contact avec le contenu du mbumba 
et noue une cordelette autour du pot tout en prononcant des paroles de conjuration. Le pot est généralement caché dans un endroit connu du seul spécialiste, souvent enterré sous les racines de l'arbre 1olo. Dans ce dernier cas, la cordelette est nouée autour de l'arbre. Le spécialiste confie au consultant un petit paquet de feuilles réputées éloigner les dangers (tiges de zika zika), calmer les situations (feuilles de lemba mpumbu) ou attirer la chance (feuilles ou tiges de nsanga valu).

La consultation des minkisi divinatoires se présente comme une recherche des causes. Les petits nkisi sont généralement utilisés pour déterminer les causes des maladies et s'inscrivent dans un processus thérapeutique conduisant à la guérison; les grands entrent dans le cadre d'une procédure socio-juridique consistant à rechercher le coupable (kanga mengo), procédure pouvant aboutir à une ordalie ${ }^{8}$. Dans ce cas, la statue est portée par le spécialiste, lors d'une séance d'invocations accompagnée de chants et de danses, afin qu'elle puisse désigner le coupable.

Les minkisi imprécatoires les plus connus sont ceux que la littérature sur l'art africain traditionnel appelle "fétiches à clous", qui autrefois servaient à la malédiction et à l'envoûtement, c'est-à-dire à l'exercice d'une vengeance. Longtemps les auteurs ont été impuissants à rendre compte de ces statues hérissées de lames, de couteaux et de clous; certains ont parlé de "culte des divinités naturelles", d'autres y ont vu une influence de "I'iconographie chrétienne, celle du Christ martyr"9.

En réalité on ne peut comprendre la coutume d'enfoncer des objets pointus dans des statues que si on la replace dans une pratique sociale courante au début du siècle. Cette pratique consistait, lors d'une situation désespérée, à "provoquer" la colère d'une personne puissante afin que celle-ci en prenne prétexte pour se venger. La pratique était courante pour obtenir une mise en servitude volontaire : toute personne qui se sentait menacée pouvait se mettre sous la dépendance et la protection d'un plus puissant en s'en

${ }^{8}$ Cette cérémonie ne se fait plus depuis longtemps.

9Voir à ce sujet Delange, 1967:137-138, et Van Buick, 1935. 
prenant soit à sa personne, soit à ses biens (bula mbunga, briser le gobelet) puisque, ne pouvant pas réparer le tort, la seule solution était que le coupable aliène sa personne (A. Belcommune, 1922:46). De même tout individu lésé dans sa personne ou ses droits (vol, calomnie, insulte, haine...) pouvait s'adresser à une force surnaturelle pour être vengé, "en provoquant de la douleur" dans la représentation matérielle de la force et en arguant que la responsabilité incombe à celui qui a porté préjudice au plaignant 10 . Cette opération (banda zimbawu, "enfoncer la vengeance") s'adressait à toutes les forces surnaturelles. Ainsi si l'on souhaitait l'intervention d'un esprit de la terre, il suffisait de lancer des imprécations en frappant le sol de la main ou en y enfonçant un morceau de bois (on parle aussi en ce cas de koma mianda). Si l'on veut être vengé par un génie simbi ou par une autre force surnaturelle, il suffit de nouer (lukuvi) une ficelle autour d'un objet qui lui est dédié en même temps qu'on maudit I'auteur supposé du préjudice. Pour déclencher l'action de rétorsion, d'autres minkisi, comme Mpizi par exemple, doivent être frappés avec des branches épineuses ou des poivriers.

Les forces surnaturelles les plus sollicitées étaient celles qui étaient représentées par des statues imprécatoires que les collectionneurs ont retrouvées toutes hérissées de clous et de lames. Cette préférence vient du fait qu'on croyait qu'elles ne lâchaient jamais les victimes qu'on leur proposait et que leur action était impitoyable, d'où leur aspect terrifiant et leurs noms frappant l'imagination afin d'inspirer la terreur aux malfaiteurs. Le consultant devait y enfoncer un clou, pour provoquer de la douleur au nkisi et donc une vengeance certaine. Autrefois on utilisait des épines dures, la statue étant souvent en bois léger. Les imprécations devaient préciser la nature de la punition souhaitée en fonction de la gravité du préjudice et des domaines d'action du nkisi. Mavungu par exemple provoquait des évanouissements; Mabiala et Mangaka de

${ }^{10} \mathrm{Ce}$ qui diffère de la magie européenne dans laquelle le magicien s'attaque à la victime par l'intermédiaire d'un objet qui la représente. 
graves maux de tête, Kozo, la folie ${ }^{11}$. Le consultant du nkisi loko enfonçait le clou à moitié pour faire souffrir la victime ou les membres de sa famille le plus longtemps possible ; il le pliait pour obtenir leur mort. Ajoutons que ces clous étaient aussi enfoncés dans les trones de baobab (nkondo, le plus célèbre nkondo kuta se trouve à Nsinda près de Tshiowa au Cabinda) pour que la victime meure.

c. Les conséquences d'un acte d'envoûtement

La pratique de mbawu a plusieurs implications. Elle peut être considérée comme une simple opération économique permettant au propriétaire du nkisi de faire des profits (Phillips, 1887:160)12. Aucune responsabilité morale ne lui est pratiquement imputée. Le nkisi ne poursuit que les coupables et non les innocents. Cependant le propriétaire d'un nkisi mbumba ou loko est suspect de sorcellerie parce que sa confection nécessite le sacrifice d'une ou plusieurs personnes dont le principe vital (monyo muntu ou tshinya) doit activer le nkisi.

Pour celui qui sollicite l'intervention d'un nkisi, la pratique du mbawu n'est pas non plus réprouvée parce qu'elle s'inscrit dans une logique de vengeance fondée sur le droit de poursuite. Il lui est cependant recommandé d'être sûr du bien-fondé des motivations du mbawu et de ne pas dépasser la mesure en ce qui concerne la sanction souhaitée sous peine de subir les mêmes châtiments.

On suspecte un envoutement par le mbawu lorsque les malheurs se succèdent, lorsqu'une maladie traîne en longueur, lorsque surviennent des accidents inattendus ou encore des disparitions fréquentes et inexplicables parmi les proches. Le recours à une ou plusieurs techniques de divination permet de déterminer la nature et l'origine de l'agression (envoûtement) ainsi que les moyens d'y mettre fin. La connaissance exacte des raisons qui ont motivé la mise en action

${ }^{11}$ Il n'existe pratiquement plus de minkisi à clous. Nous avons par contre trouvé à Kinshasa des statues-bustes, utilisées dans le même but, avec des cadenas fermés, signifiant la fermeture de la chance et/ou de l'avenir de la victime.

12 Philips évalue le coût de l'extraction d'un clou à 10 livres et plus vers les années 1880 . 
d'un mbawu est importante pour éviter que d'autres membres du clan n'en soient la victime, puisqu'il est dit que les effets du mbawu ne cessent de se manifester que lorsque son auteur a été dédommagé et que des rituels appropriés ont été accomplis.

En effet, les causes du mbawu ayant été déterminées, la victime, ou plus souvent un représentant de son clan ou un porte-parole (nkoto kwanda), prend contact avec le propriétaire du nkisi en vue de suspendre provisoirement les effets du mbawu (fuka zimbawu). Cette suspension permet la négociation du dédommagement; dès que celle-ci aboutit, elle anihile le mbawu (kula zimbawu, délivrer les mbawu; vonza zimbawu, annihiler les envoûtements). Ce rite est accompli auprès du prêtre d'un esprit de la terre (ntoma si) pour les mbawu za ndombe (envoûtements dans le sanctuaire), auprès d'un responsable des rites d'un esprit particulier pour les mbawu za simbi, ou auprès du propriétaire du nkisi dans lequel a été enfoncé un clou pour les mbawu zi seende. Dans les deux premiers cas, le spécialiste du culte procède à la purification de la victime et des membres de son clan avec de l'eau lustrale. Il préside aussi à un repas collectif où ensemble ils consomment de la viande d'une chèvre sacrifiée à cet effet.

La conjuration de l'envoûtement par les mbawu zi seende exigeait autrefois la présence de tous les membres du clan, les absents ainsi que les enfants dont la naissance est attendue devant être représentés par des tiges de bois. Le rituel commençait par des suppliques ; le sang d'une chèvre était ensuite versé sur le sol et l'on retirait le clou (sumuna, désamorcer ; sonuna seende, lâcher le clou ou botula seende, retirer le clou) préalablement identifié par le devin comme étant celui qui avait servi à l'envoûtement. Tous les membres du clan étaient enfin purifiés par l'eau lustrale.

\section{Les minkisi et la sorcellerie}

Nous avons vu à plusieurs reprises que la détention d'un nkisi pouvait conduire à des actes de sorcellerie. Celle-ci consiste à accaparer, dans des circonstances mystérieuses, la substance vitale d'un individu dans des buts inavouables et égoìstes. Les Woyo pensent 
que les sorciers forment une association qui tient régulièrement des réunions dans les arbres situés près des carrefours ou des marchés, rassemblements au cours desquels ils dévorent les victimes qu'ils fournissent à tour de rôle. Il va sans dire que la dénonciation d'un sorcier peut conduire à un conflit généralisé à l'intérieur du clan, voire même à son éclatement. Si par contre l'action du sorcier se révèle avoir fait des victimes à l'extérieur du clan, celui-ci se trouve solidairement en situation de "dette" (sombe tshya mesa), ce qui autrefois, pouvait provoquer sa réduction à l'esclavage. Certains croient que cette forme de sorcellerie est innée et se matérialise par la présence de sachets (makundu) près du coeur ou par une excroissance (ndokila) au niveau de l'estomac ${ }^{13}$. D'autres pensent au contraire qu'on peut devenir sorcier en fréquentant sans le savoir des personnes réputées telles ou en prenant part par inadvertance à une rencontre de sorciers.

La capture par les sorciers se ferait de façon mystérieuse; elle se déroule dans un monde invisible parallèle au monde réel. Grâce à son pouvoir mystique, le sorcier est censé agir à partir d'un lieu liminaire appelé ku bwilu (lieu obscur, nocturne) et ku nyimbi (lieu ténébreux); ce lieu serait une sorte de représentation en creux du monde réel, visible (ku mwinyi, lieu clair). Cette réplique du monde visible lui serait solidaire, en sorte qu'un événement qui a lieu dans l'un a son correspondant dans l'autre, avec des conséquences égales dans les deux univers. Le sorcier se déplacerait sans être vu dans ces deux univers et agirait tantôt dans l'un tantôt dans l'autre. Ses actions maléfiques consistent à piéger ou à attaquer ouvertement ses victimes. Le sorcier conduirait ses victimes dans l'"au-delà" (buka) représenté par des lieux désolés comme les cimetières, les gouffres, le creux des arbres ou des rochers, ou les endroits désertiques. Au buka, les victimes seraient installées dans des sortes de villages où le sorcier règne en mâtre et seraient utilisées au fur et à mesure à ses propres fins. On croit aussi que certaines victimes sont placées dans des navires "mystiques" (kumbi la paanya)

${ }^{13}$ Il n'y aurait, semble-t-il, pas de rapport entre ces excroissances et les médecines introduites dans la partie ventrale du nkisi. 
ou dans des camps de travail (tshiungu) où des populations d'esclaves à la merci du sorcier vivraient sous formes de zombi 14 , travaillant sans relâche pour accroître la richesse de leur mâtre.

Le système des minkisi peut être associé à la sorcellerie pour deux raisons : soit que le propriétaire du nkisi cherche à renforcer sa puissance, soit que les intentions du consultant soient considérés comme anti-sociales. Nous avons vu que l'initiative de ce dernier s'inscrit dans une logique de vengeance admise par la société ; contrairement à la sorcellerie qui agirait de manière insidieuse et inéluctable, les effets du nkisi ne sont pas fatals, puisqu'on peut les arrêter par un dédommagement équitable et une purification rituelle appropriée.

Rappelons que l'acquisition des deux catégories de minkisi, mbumba et loko, exigent du futur propriétaire de l'objet le sacrifice d'une ou de plusieurs personnes dont le principe vital doit animer le nkisi. Il en va de même lorsque le nganga doit renouveler (sukumuna) la puissance de son nkisi ou lorsque, constatant une défaillance dans son efficacité, il doit le "revivifier" (fundulula mafwanga fwanga). A la différence du sorcier, il semble que le détenteur du nkisi prélève une victime dans son clan dans l'intérêt de son groupe, pour le protéger contre les attaques extérieures ou pour acquérir une clientèle capable de relever son prestige. La possession d'un nkisi étant réservée à des personnalités du clan et les victimes étant choisies parmi les éléments marginaux de ce groupe, il est clair que le système du nkisi renforce l'ordre et la cohésion du groupe autour de l'autorité du chef de clan. Aussi ce prélèvement de vies humaines peut-il être considéré comme une sorcellerie "licite" (L. de Heusch, 1971: 173-174). Elle est néanmoins regardée comme anti-sociale et condamnable si les membres de la section de clan dont fait partie la victime se sentent lésés et contestent l'autorité et la bonne foi du propriétaire du nkisi,

14 Nous retrouvons une conception similaire chez les Douala (Rosny, 1981: 89-101). Comme le suggère cet auteur, il faut voir dans cette conception une réminiscence de la traite des esclaves. Quant au mot zombi, Merolla da Sorento (1702) attribue son origine aux Woyo et aux Kakongo; il signifierait "an apparition of deceased person". 
ce qui peut aboutir à la scission du clan 15 ; ou encore si, pour épargner un membre du clan, l'acquisition, l'entretien et la réactivation de la force du nkisi ont été obtenus en captant l'âme d'un être étranger au clan (homme ou esprit errant). Ce dernier cas peut donner lieu à de sérieuses accusations dans lesquelles l'ensemble du clan se trouve en situation de débiteur (sombe tshya bwilu, dette de la nuit); le groupe doit alors céder en compensation une personne d'un statut équivalent à celui de la victime ainsi que de nombreux biens. On peut donc parler d'une forme de sorcellerie qui est certes juridiquement repréhensible mais pas de la même façon que la sorcellerie ordinaire, qui, rappelons-le, entrainait lorsqu'elle était identifiée comme telle, la mise à mort du sorcier.

Concluons en constatant que le terme nkisi s'applique à diverses catégories d'objets qui s'inscrivent dans un système global de représentations exprimant des rapports entre l'homme et le surnaturel d'une part, entre les hommes d'autre part. Le langage symbolique véhiculé par les minkisi n'est compréhensible que si on examine le sens accordé à chacun des éléments qui leur sont rattachés. Chez les Woyo, le nkisi est conçu comme une force indéterminée et ambivalente dont l'action, déterminée lors de son acquisition, est censée se mettre en branle chaque fois qu'un consultant la sollicite. Certains minkisi, animés par une présence humaine sont capables d'agression, basculent dans la sphère de la sorcellerie. L'usage d'un nkisi ne peut cependant être confondu avec la sorcellerie car, non seulement il annule l'agression, mais encore sa confection n'est pas guidée par un dessein égoiste et les effets de son action ne sont pas en principe fatals. Le système de représentations associé aux minkisi est, enfin, socialement important car il permet la mâtrise des données négatives de la société : le nkisi paraît être ainsi l'expression nécessaire des désarrois existentiels, des frustrations et des espoirs déçus.

Mulinda Habi Buganza

Institut des Musées Nationaux du Zaïre

${ }^{15}$ Les accusations de sorcellerie dévoilent souvent l'existence de conflits entre les personnalités du clan. On voit ainsi nâtre des alliances dans lesquelles le groupe paternel (tshitaata) prend publiquement la défense des "fils", en dénonçant les abus du groupe maternel (thimaama). Voir à ce sujet Nguvulu, 1971: 30. 


\section{Bibliographie}

Bassani E.

1977 "Kongo Nail Fetishes from the Chiloango River Area", African Arts, $X, 3$.

Belcommune A.

1922 Vingt années de vie africaine : récits de voyage, d'aventures et d'exploration au Congo Belge, 1874-1893, Vve F. Lancier, Bruxelles.

Bittremieux L.

1934 "Symbolism in Negerkunst", Congo, V, 2.

Buakasa Tulu K.M.

1973 L'impensé du discours : kindoki et nkisi en pays Kongo du Zaïre, P.U.Z., C.E.D.A., Kinshasa/Bruxelles.

Delange J .

1967 Arts et Peuples de 1'Afrique Noire. Introduction à une analyse des créations plastiques, Gallimard, Paris.

Dennett R.

1887 Seven Years among the Fjort, being an English trader's experience in the Congo District, Londres.

1901/"The religion of the Fyort or Fiote Mavungo", Journal of

1902 the African Society, pp.452-454.

Doutreloux A.

1967 L'ombre des fétiches : société et culture Yombe, Nauwelaerts,

Paris.

Dupré M.C.

1975 "Les systèmes des forces nkisi chez les Kongo, d'après le $3 e$ volume de K. Laman", Africa, XLV, 1, 12-28.

Hagenbucher-Sacripanti

1973 Les fondements spirituels du pouvoir au royaume de Loango, ORSTOM, Paris.

Heusch L. de

1971 Pourquoi 1 'épouser? et autres essais, Gallimard, Paris.

Hirschberg $W$.

1971 "Gedanken um einen Spiegelfetish", Ethnologische Zeitschrift, I, 41-46.

Jacobson Widding $A$.

1979 "Red-White-Black as mode of thought : a study of triadic classification by colours in the ritual symbolism and cognitive thought of people of Lower Congo", Acta universitatis Upsalensis, 1, Umqvist \& Wiksell International, Upsala, Stockholm. 
Mac Gaffey W.

1977 "Fetishism revisited : kongo nkisi in sociological perspective", Africa, XLVII, 2, 172-184.

Mac Gaffey W. \& Janzen J.M.

1974 "Nkisi figures of Bakongo", African Arts VII, 3, 87-89.

Mattos e Silva J. de

1904 Contribuiçao para estudio da regiao de Cabinda, Tipografia universal, Lisboa.

Merolla da Sorento J.

1702 A Voyage to Congo and several other countries, in Collection of voyages and travels, ed. A. Churchill, Londres.

Nguvulu

1971 L'humanisme néo-africain face au développement, Okapi, Kinshasa.

Philips R.C.

1887 "The Social System of the Lower Congo", The journal of the Manchester geographical Society, III.

Rosny E. de

1981 Les yeux de ma chèvre, Terre Humaine, Paris.

Rouvre Ch. de

1880 "La Guinée méridionale indépendante, 1870-1877", Bulletin de la Société de Géographie de Paris.

Van Buick

1935 "Notes d'ethnologie : style oral et symbolisme au Mayombe", Congo, I, 5.

Volavka Z.

1972 "Nkisi Figures of Lower Congo", African Arts, V, 2, 52-59.

1974 "Once Again on the Kongo nkisi figures", African Arts, VII, 3, 89-90. 